\title{
Preliminary Characterization of a Homogeneous Polysaccharide with Anticomplement Activity from Sijunzi Decoction
}

\author{
Ruijun Wang $(\mathbb{D}$, Yunfei Ji $\mathbb{D}$, Ying Peng $(\mathbb{D}$, and Xiaobo Li $\mathbb{C}$ \\ School of Pharmacy, Shanghai Jiao Tong University, Shanghai 200240, China \\ Correspondence should be addressed to Xiaobo Li; xbli@sjtu.edu.cn
}

Received 27 August 2017; Revised 14 November 2017; Accepted 5 December 2017; Published 16 January 2018

Academic Editor: Wieslaw Wiczkowski

Copyright (C) 2018 Ruijun Wang et al. This is an open access article distributed under the Creative Commons Attribution License, which permits unrestricted use, distribution, and reproduction in any medium, provided the original work is properly cited.

\begin{abstract}
Sijunzi decoction (SJZD) is a classical herbal prescription in traditional Chinese medicine (TCM) used for enhancing the function of immune system. In previous studies, a polysaccharide fraction S-3 was screened from SJZD by assessment of immune system regulation, intestinal microbiota, and SCFA in order to explore the immune active ingredients in SJZD. In the present study, S-3 was further purified, and a homogeneous polysaccharide S-3-1 with a molecular mass of $13.5 \times 10^{4}$ Da was obtained after further fractionation by Sephadex G-150 size-exclusion chromatography. The immunological activities of S-3-1 were assayed in vitro for the first time. The determination of the anticomplement activity showed that S-3-1 displayed inhibitory effects on classical pathway of the complement system, with $\mathrm{CH}_{50}$ values of $530 \mu \mathrm{g} / \mathrm{mL}$. The FT-IR analysis showed that S-3-1 had absorptive peaks characteristic of polysaccharides. The methylation and GC-MS analysis showed that it is comprised of Rha, Ara, Xyl, Man, Gal, and Glc in a relative molar ratio of $0.35: 0.37: 1.4: 0.31: 3: 0.8$ and that it mainly contained 1,4-linked-Glc and 1,6-linked-Gal glycosidic bonds. The morphology of S-3-1 was observed by atomic force microscope (AFM). These results provided evidences for tracking the material basis of SJZD immune activity.
\end{abstract}

\section{Introduction}

Sijunzi decoction (SJZD) is a classic prescription used in traditional Chinese medicine (TCM) and is prepared using four kinds of Chinese herbs, Ginseng Radix, Atractylodes Macrocephalae Rhizoma, Poria, and Glycyrrhizae Radix. It is well known for curing spleen deficiency $[1,2]$, characterized by the comprehensive manifestation of diseases in multiple systems, such as the digestive (digestion, absorption energy conversion) and the immune system. Modern pharmacological studies have shown that SJZD prescription affects the above physiological functions by strengthening the immune system [3]. Although biologically active, low molecular mass compounds from SJZD such as flavone and saponin have previously been studied, they do not account for all of the components in SJZD. Polysaccharide is the most abundant and main effective component in SJZD [4]. Polysaccharide extracted from SJZD is an effective immunomodulator and immune adjuvant [5]. There are also reports of other TCM that contain polysaccharides and include Ginseng Radix [6],
Atractylodes Macrocephalae Rhizoma [7], Poria [8], and Glycyrrhizae Radix [9], all of which are components of SJZD. Moreover, it has now been proven that the physiological/biological effects of polysaccharides realized through TCM prescriptions are considerably better than the effects that come from single herb prescriptions [10].

There is limited data currently available on the characterization of polysaccharides from SJZD prescriptions. Recent research has detected the presence of an active polysaccharide fraction (S-3) in SJZD that can improve the immune function of intestinal endothelial lymphocytes [11]. However, information on the conformation of this polysaccharide fraction S-3 is limited. In this study therefore, we isolated and purified a new water-soluble polysaccharide from the S3 fraction of SJZD prescription, characterized its structure, and investigated the potential activity of this polysaccharide against classical complement pathways. The results of this study will offer a theoretical basis for further investigation on the structure-activity relationship and development of SJZD polysaccharides. 


\section{Materials and Methods}

2.1. Herbal Medicine and Chemicals. SIZD was prepared by combining four herbs, Ginseng Radix, Atractylodes Macrocephalae Rhizoma, Poria, and Glycyrrhizae Radix, in the ratio of $3: 3: 3: 2$ by weight. The herbs were bought from Shanghai Huayu Pharmaceutical Co., Ltd. (Shanghai, China), their botanical origins were identified by the corresponding author, and voucher specimens were deposited at the School of Pharmacy, Shanghai Jiao Tong University.

Trifluoroacetic acid (TFA), dextrans, and monosaccharide standards were purchased from Aladdin Reagent Int. (Shanghai, China). All other chemicals used were of analytical grade as available.

2.2. Extraction and Purification of Polysaccharide from SJZD. SJZPS (crude SJZD polysaccharide) and polysaccharide fraction S-3 were prepared as described previously [11]. S-3 was further purified by gel filtration chromatography on a Sephadex G-150 column $(16 \mathrm{~mm} \times 70 \mathrm{~cm})$ and eluted with deionized water at flow rate $30 \mathrm{~mL} / \mathrm{h}$. Two polysaccharides were obtained through elution, named S-3-1 and S-3-2, and the yield was $24.5 \%$ and $1.2 \%$, respectively.

\subsection{Determination of Molecular Mass and Analysis of Monosaccharide Composition of Polysaccharides}

2.3.1. Determination of Homogeneity and Relative Molecular Mass. The molecular mass of S-3-1 and S-3-2 were determined by high performance gel permeation chromatography (HPGPC) on a TSK-GEL G4000PWxl column $(7.5 \times 300 \mathrm{~mm}$, Tosoh Co., Japan) as described previously [12]. Deionized water was used as the elution and flow rate was maintained at $1 \mathrm{~mL} / \mathrm{min}$. A $20 \mu \mathrm{L}$ aliquot was injected for each run. The calibration curve of $\log (\mathrm{Mw})$ versus elution time $(T)$ is given by $\log (\mathrm{Mw})=-0.1264 T+5.6361$.

2.3.2. General Analysis Methods. Carbohydrate content of the polysaccharide was determined by phenol-sulfuric acid colorimetric methods [13] using D-Glc as the standard. Protein content was measured by the Lowry method [14] using BSA as the standard. Uronic acid content was measured by photometry with m-hydroxydiphenyl at $525 \mathrm{~nm}$ using dgalacturonic acid as the standard [15].

2.3.3. Monosaccharide Composition Analysis. Monosaccharide composition analyses of S-3-1 and S-3-2 were performed by GC-MS (model 7890/5975C-GC/MSD, Agilent Technologies; Santa Clara, CA, USA) as previously described [16]. $10 \mathrm{mg}$ of each sample was hydrolyzed with $2 \mathrm{~mol} / \mathrm{L}$ trifluoroacetic acid at $95^{\circ} \mathrm{C}$ for $2 \mathrm{~h}$. The excess acid was removed by four successive washes with methyl alcohol. For GC-MS analysis, the complete hydrolysate was mixed with $5 \mathrm{mg}$ hydroxylamine hydrochloride, $0.5 \mathrm{~mL}$ pyridine, and $4 \mathrm{mg}$ inositol as the internal standard and incubated at $90^{\circ} \mathrm{C}$ for $30 \mathrm{~min}$. The mixture was cooled to room temperature and $0.5 \mathrm{~mL}$ acetic anhydride was added, mixed thoroughly, and incubated at $90^{\circ} \mathrm{C}$ for $30 \mathrm{~min}$. After cooling, the resulting aldononitrile acetate derivatives were analyzed by GCMS. Aldononitrile acetate derivatives of seven authentic monosaccharide standards with inositol as internal standard were prepared and subjected to GC-MS analysis the same way. By comparison based on retention times, monosaccharide compositions of S-3-1 and S-3-2 were determined.

\subsection{Structural Characterization of S-3-1}

2.4.1. UV and IR Spectra Analysis. The impurity content in polysaccharide was determined by ultraviolet spectra analysis. Absorbance of wavelengths in the 200-400 nm regions was recorded with a UV spectrophotometer. The organic functional groups of polysaccharide were identified by Fourier transform infrared (FT-IR) method. Polysaccharide $(2 \mathrm{mg})$ was dried at $35-45^{\circ} \mathrm{C}$ in vacuum over $\mathrm{P}_{2} \mathrm{O}_{5}$ for $48 \mathrm{~h}$ prior to grinding with spectroscopic grade $\mathrm{KBr}$ powder and then pressed into a $1 \mathrm{~mm}$ pellet for FT-IR measurement by a Thermo Fisher Nicolet 6700 FT-IR spectrometer [17].

2.4.2. Periodate Oxidation and Smith Degradation. S-3-1 sample $(25 \mathrm{mg})$ was oxidized with $15 \mathrm{mM} \mathrm{NaIO}_{4}(25 \mathrm{~mL})$ and kept in the dark at $4^{\circ} \mathrm{C}$. At $12 \mathrm{~h}$ intervals, $30 \mu \mathrm{L}$ aliquots were taken, diluted 250 times with distilled water, and read by spectrophotometry at $223 \mathrm{~nm}$ [18]. The amount of $\mathrm{NaIO}_{4}$ consumed was calculated according to the $\mathrm{NaIO}_{4}$ standard curve. Production of formic acid was determined by titration. The remaining periodate product was treated with ethylene glycol and dialyzed for $24 \mathrm{~h}$. The content inside the dialysis sack was concentrated and reduced with $\mathrm{NaBH}_{4}$ overnight, and $\mathrm{pH}$ was adjusted to 5.5-7.0 with $0.1 \mathrm{~mol} / \mathrm{L}$ acetic acid and further dialyzed against deionized water for $48 \mathrm{~h}$. The dialysate was collected and concentrated to dryness by rotary evaporation at $50^{\circ} \mathrm{C}$. Then $4.0 \mathrm{~mL}$ methanol was added and evaporated to dryness for four times. The resulting product was hydrolyzed with trifluoroacetic acid and aldononitrile acetate derivatives were prepared by procedures described in previous section and subjected to GC-MS analysis.

2.4.3. Methylation Analysis. Methylation of S-3-1 sample was performed according to the method of Ciucanu and Kerek [19], with minor modifications. An S-3-1 sample was methylated with $\mathrm{CH}_{3} \mathrm{I}$ in dimethyl sulfoxide in the presence of dry sodium hydroxide. $2 \mathrm{~mL}$ distilled water was added to stop the reaction and the methylated sample was then extracted with $2 \mathrm{~mL}$ methyl dichloride. The methylene chloride extract was separated by centrifugation and evaporation. The disappearance of the $\mathrm{OH}$ band $\left(3200 \mathrm{~cm}^{-1}\right.$ to $\left.3700 \mathrm{~cm}^{-1}\right)$ in FTIR spectrum was used to confirm complete methylation [18]. Methyl derivatives were prepared subsequently as follows: hydrolyzing the premethylated polysaccharide sample by treatment with $88 \%$ formic acid solution $(3 \mathrm{ml})$ at $100^{\circ} \mathrm{C}$ for $3 \mathrm{~h}$ and $2 \mathrm{M} \mathrm{TFA}(3 \mathrm{~mL})$ at $100^{\circ} \mathrm{C}$ for $6 \mathrm{~h}$. The hydrolysate was reduced with sodium borodeuteride $\left(\mathrm{NaBH}_{4}\right)$ solution $\left(5 \%\right.$ in water) at room temperature $\left(25^{\circ} \mathrm{C}\right)$ for $4 \mathrm{~h}$ and acetylation with acetic anhydride $\left(\mathrm{Ac}_{2} \mathrm{O}\right)$ at $100^{\circ} \mathrm{C}$ for $1 \mathrm{~h}$. The resulting methylated derivatives were detected by GC-MS 

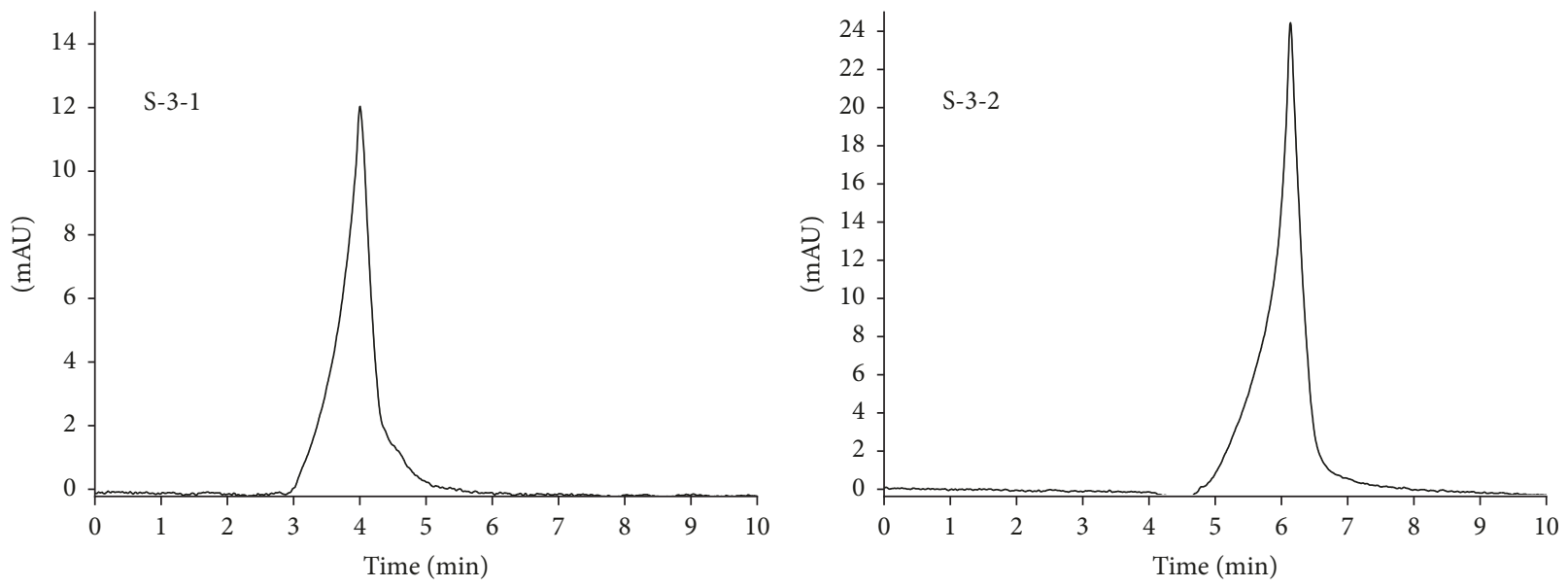

FIGURE 1: HPGPC profile of polysaccharide S-3-1 and S-3-2.

(model 7890/5975C-GC/MSD, Agilent Technologies; Santa Clara, CA, USA).

2.4.4. NMR Analysis. Dried polysaccharide sample $(20 \mathrm{mg})$ was dissolved in $1 \mathrm{~mL}$ of $\mathrm{D}_{2} \mathrm{O}$ and kept at room temperature for $3 \mathrm{~h} .{ }^{13} \mathrm{C}$ and ${ }^{1} \mathrm{H}$ NMR spectra were recorded with a Bruker AMX-600 NMR spectrometer using sodium trimethylsilyl propionate (TSP) as internal standard, $\mathrm{D}_{2} \mathrm{O}$ as the solvent at $25^{\circ} \mathrm{C}$, and the HOD signal fixed at $4.79 \mathrm{ppm}$.

2.4.5. Atomic Force Microscopy. The molecular morphology of S-3-1 in solution was observed using a Nanonavi ESweep Environment Control Scanning Probe Microscope [20] (Seiko, Japanese). The polysaccharide $(50 \mu \mathrm{g} / \mathrm{mL})$ was dissolved in deionized water with gentle stirring and serially diluted to $10 \mathrm{ng} / \mathrm{mL}$. Next, a drop of $5 \mu \mathrm{L}$ polysaccharide solution was placed on the mica and dried at $25^{\circ} \mathrm{C}$ for at least $1 \mathrm{~h}$. Imaging of samples was performed by the microscope in tapping mode. Nanoscope software was used to obtain and process AFM images.

2.5. Complement-Fixing Assay. The complement-fixing assay was performed as previously described [21, 22]. Antibodysensitized sheep erythrocytes were washed three times with BBS containing $2.8 \mathrm{mM}$ barbitone sodium and $0.8 \mathrm{mM}$ barbital before use. The erythrocytes were prepared to a concentration of $2 \%$ cell suspension in BBS and used the same day. Guinea pig serum was diluted with BBS to a concentration giving about $50 \%$ hemolysis. Triplicate samples containing $50 \mu \mathrm{L}$ of each serially diluted polysaccharide fraction were mixed with $50 \mu \mathrm{L}$ diluted serum, added to microplate wells, and incubated at $37^{\circ} \mathrm{C}$. After $10 \mathrm{~min}$, sensitized sheep erythrocytes $(100 \mu \mathrm{L})$ and BBS $(100 \mu \mathrm{L})$ were added to each well, and the samples were incubated for an additional $30 \mathrm{~min}$ at $37^{\circ} \mathrm{C}$. After centrifugation ( $2152 \mathrm{~g}$ for $10 \mathrm{~min}$ ), $200 \mu \mathrm{L}$ of each supernatant was added in flat-bottom microplates, and absorbance was measured at $405 \mathrm{~nm}$. 100\% lysis was obtained by adding distilled $\mathrm{H}_{2} \mathrm{O}$ to sensitized sheep erythrocytes. Samples containing BBS, serum, and sensitized sheep erythrocytes were used as background controls $\left(A_{\text {control }}\right)$, while heparin served as a positive control. Inhibition of hemolysis induced by the test samples was calculated by the formula: $\left[\left(A_{\text {control }}-A_{\text {sample }}\right) / A_{\text {control }}\right] \times 100 \%$. A doseresponse curve (6-7 points) was constructed to calculate the concentration of test sample able to give $50 \%$ inhibition of hemolysis $\left(\mathrm{CH}_{50}\right)$. A low $\mathrm{CH}_{50}$ meant high complementfixing activity.

\section{Results}

3.1. Molecular Mass and Chemical Composition Analysis of Homogeneous Polysaccharides Purified from S-3. Fractionation of polysaccharides S-3-1 and S-3-2 by Sephadex G150 size-exclusion chromatography resulted in a HPGPC profile as shown in Figure 1. The single, symmetrically sharp peak showed by each polysaccharide in the HPGPC profile revealed that they were homogeneous polysaccharides. Based on the retention times of each polysaccharide, average molecular mass of S-3-1 and S-3-2 was estimated to be $13.5 \times 10^{4} \mathrm{Da}$ and $7.1 \times 10^{4} \mathrm{Da}$, respectively.

The total sugar content of S-3-1 was higher (99.67\%) than that of S-3-2 (97.42\%). No protein content was detected in S-3-1 and S-3-2. The uronic acid content in the two polysaccharides was found to be $1.03 \%$ and $6.25 \%$, respectively, suggesting that S-3-2 belonged to the class of acidic polysaccharides.

Alditol acetates of each polysaccharide hydrolysate were subjected to GC-MS analysis. The neutral monosaccharide composition of S-3-1, based on retention times and peak areas, was determined to be Rha, Ara, Xyl, Man, Gal, and Glc in molar ratio $0.35: 0.37: 1.4: 0.31: 3: 0.8$. The monosaccharide composition of S-3-2 was determined to be Rha, Ara, Xyl, Man, Gal, and Glc in molar ratio $0.75: 0.24: 1.2: 0.17: 0.89: 0.23$. Thus, it was concluded that $\mathrm{S}$ 3-1 and S-3-2 are heteropolysaccharides with the same kind of monosaccharide composition.

3.2. Structural Characterization of S-3-1. In the ultraviolet spectra analysis S-3-1 did not display significant absorption 
TABLE 1: Results of periodate oxidation/Smith degradation of S-3-1.

\begin{tabular}{lccc}
\hline Linkage & $\begin{array}{c}\text { Consumption of periodate } \\
(\mathrm{mol} / \mathrm{mol} \mathrm{Glc})\end{array}$ & $\begin{array}{c}\text { Yield of formic acid } \\
(\mathrm{mol} / \mathrm{mol} \mathrm{Glc})\end{array}$ & Products of Smith degradation \\
\hline $1 \rightarrow 2$ & 1 & nd & Glycerol \\
$1 \rightarrow 3$ & nd & nd & Gal/Man \\
$1 \rightarrow 4$ & 1 & nd & Erythritol \\
$1 \rightarrow 6$ & 2 & 1 & Glycerol \\
& 1.047 & 0.4533 & Glycerol: Erythritol: Man : Gal $=5.57: 0.82: 0.60: 0.28$ \\
\hline
\end{tabular}

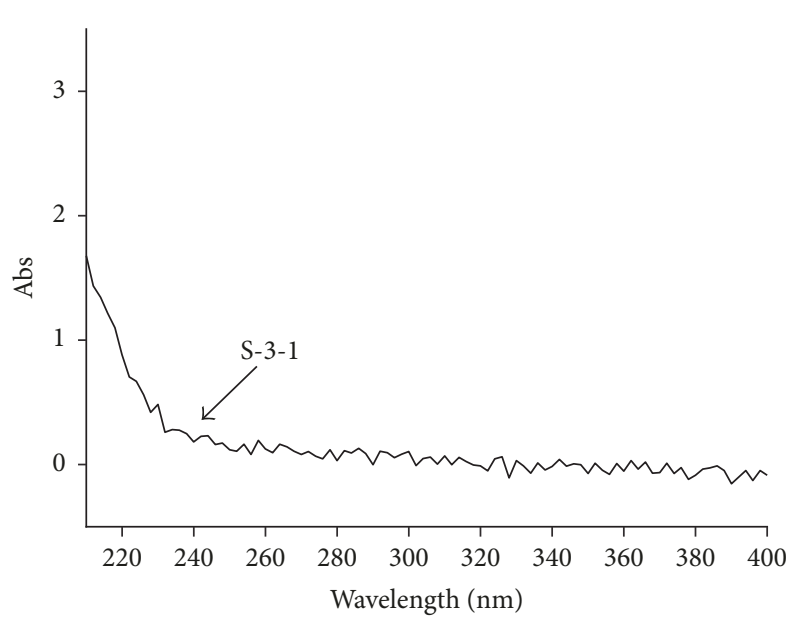

FIGURE 2: UV spectra of S-3-1.

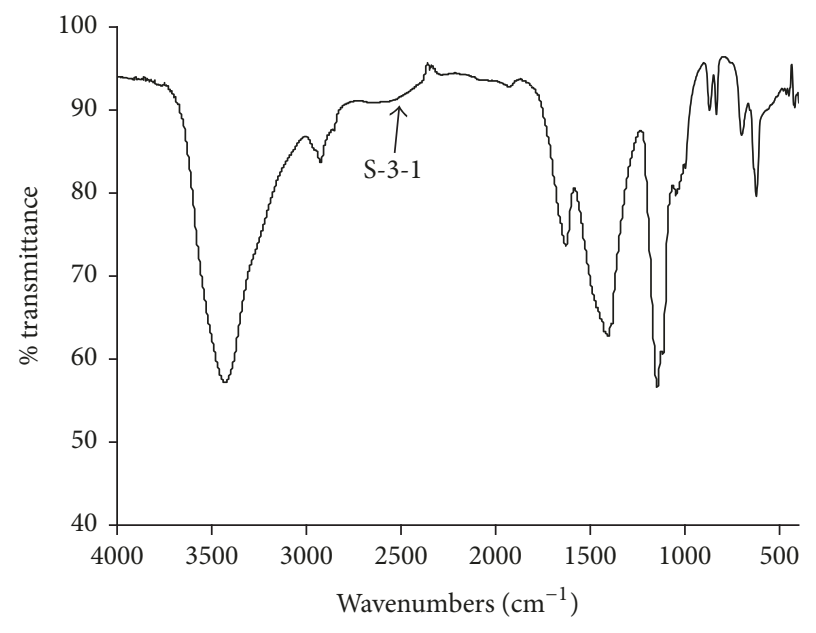

FIgURE 3: FT-IR spectra of S-3-1. peak between wavelengths $280 \mathrm{~nm}$ and $260 \mathrm{~nm}$ (Figure 2), indicating that protein and nucleic acid impurity have been reduced to a minimum. The FT-IR spectra of S-3-1 revealed polysaccharide specific absorption bands at $3600-3200 \mathrm{~cm}^{-1}$, $3000-2800 \mathrm{~cm}^{-1}$, and $1400-1200 \mathrm{~cm}^{-1}$ (Figure 3). The strong broad absorption peak at $3429-3430 \mathrm{~cm}^{-1}$ was due to the hydroxyl stretching vibration of the polysaccharide, and the peak at $2923-2920 \mathrm{~cm}^{-1}$ was due to the $\mathrm{C}-\mathrm{H}$ stretching vibration band. The peak at $1630 \mathrm{~cm}^{-1}$ was attributed to the bending vibration of $\mathrm{O}-\mathrm{H}$, and the signal at 1383$1384 \mathrm{~cm}^{-1}$ band was attributed to the stretching vibration of $\mathrm{C}-\mathrm{H}$ bending in the $\mathrm{CH}_{2}$ group $[12,23-25]$. The bands at $1148 \mathrm{~cm}^{-1}$ and $1116 \mathrm{~cm}^{-1}$ were attributed to the vibration of C-O. The weak $1028-1093 \mathrm{~cm}^{-1}$ band corresponded to C$\mathrm{O}-\mathrm{C}$ stretching in the pyranose ring. The absorption peaks at $870-860 \mathrm{~cm}^{-1}$, indicated that mannose was present [16]. The characteristic absorption band at $834 \mathrm{~cm}^{-1}$ suggested the presence of D-Glc pyranoside configurations in S-3-1 [26].

The locations of glycosidic linkages in the polysaccharides are preliminarily determined by periodate consumption and production of formic acid in periodate oxidation [27]. As shown in Table 1, results from periodate oxidation showed that, per sugar residue, $1.04 \mathrm{~mol}$ of periodate was consumed and $0.45 \mathrm{~mol}$ formic acid was produced. The production of formic acid indicated that some of the residue may exist in the pyranohexose 1-linked or 1,6-linked form. Since the amount of periodate consumed was more than twice the amount of formic acid produced, it is possible that many linkages exist in the pyranose 1,2-linked, 1,4-linked, 1,2,6-linked, 1,4,6-linked, and 1,2,4-linked forms or furanose 1-linked forms. Moreover, the ratio of the amount of monosaccharide to amount of periodate was nearly $1: 1$ for polysaccharide S-3-1. This proved that the 1,3-linked forms that do not consume periodate were either not present or few in number [28]. The periodateoxidized products of S-3-1 were further reduced, hydrolyzed, and analyzed by GC-MS. Results showed that the degradation process completely removed glucose, $\mathrm{Rha}$, and $\mathrm{Gal}$ residues and produced a large amount of glycol, methylglycol, glycerol, and erythritol, indicating that these 4 glycoside residues may exist in the 1,4-linked, 1,4,6-linked, 1,2-linked, 1,2,6linked, or 1,6-linked forms, which could be oxidized. Ratios of Smith degradation products detected by GC-MS were Glycerol : Erythritol: Man : Gal = $5.57: 0.82: 0.60: 0.28$ in S3-1. A large proportion of galactose and mannose were probably from 1,3-linkages, erythritol from $1 \rightarrow 4$ linkages, and glycerol from 1,6- and/or 1,2-linkages.

From Table 2, based on the results of the methylation analysis on S-3-1, the following conclusions can be drawn: (1) the Gal residues were present as 1,4-linked and 1,4,6linked Galp residues; (2) the Glc residues were present as terminal, 1,4-linked, and 1,6-linked Glcp residues; (3) the Rha residues were present as terminal; (4) the Man residues were present as 1,2-linked, 1,3-linked terminal, and 1,3,6linked Manp residue; (5) the Xyl residues were present as 1,5linked Xylf residue. These residues mentioned above except terminal Rhap, Glcp, and Xylf account for 93\% of total 


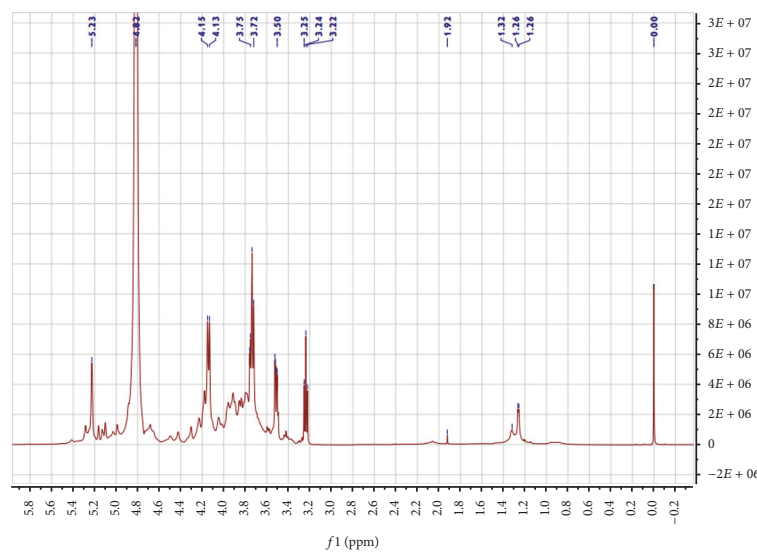

(a)

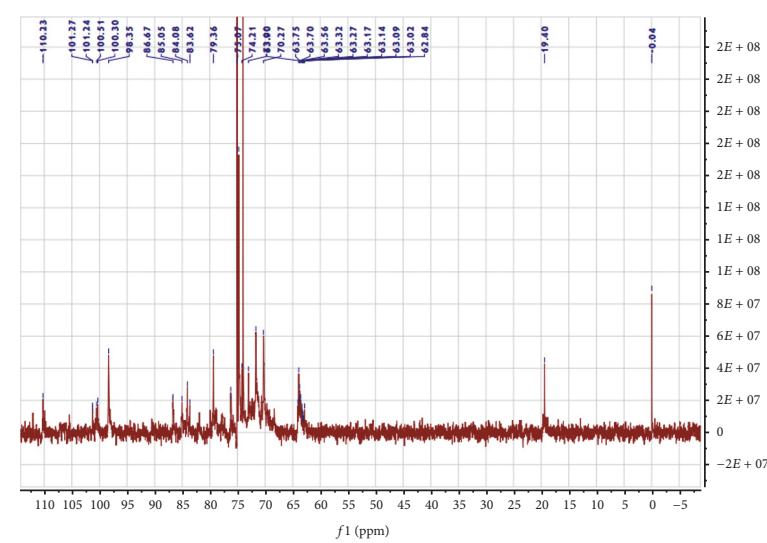

(b)

Figure 4: 600-MHz NMR spectra of S-3-1 in $\mathrm{D}_{2} \mathrm{O}$. (a) ${ }^{1} \mathrm{H}$ NMR spectrum of S-3-1. (b) ${ }^{13}$ C NMR spectrum of S-3-1.

TABLE 2: Methylation analysis of S-3-1.

\begin{tabular}{lccc}
\hline $\begin{array}{l}\text { Retention } \\
\text { time }(\mathrm{min})\end{array}$ & Linkage & Methylated sugar & Molar ratios \\
\hline 9.433 & $1 \rightarrow 6$ & $2,3,4-\mathrm{Me}_{3}-\mathrm{Glc}$ & 1.45 \\
11.036 & $1 \rightarrow 2$ & $3,4,6-\mathrm{Me}_{3}-\mathrm{Man}$ & 4.04 \\
12.909 & $1 \rightarrow$ & $2,3,4,6-\mathrm{Me}_{4}-\mathrm{Glc}$ & 2.55 \\
13.199 & $1 \rightarrow$ & $2,3,4-\mathrm{Me}_{3}-\mathrm{Rha}$ & 1.6 \\
13.385 & $1 \rightarrow 3$ & $2,4,6-\mathrm{Me}_{3}-\mathrm{Man}$ & 2.28 \\
14.287 & $1 \rightarrow 4$ & $2,3,6-\mathrm{Me}_{3}-\mathrm{Glc}$ & 9.49 \\
14.499 & $1 \rightarrow 4,6$ & $2,3-\mathrm{Me}_{2}-\mathrm{Gal}$ & 2.16 \\
14.614 & $1 \rightarrow 6$ & $2,3,4-\mathrm{Me}_{3}-\mathrm{Gal}$ & 38.3 \\
16.681 & $1 \rightarrow 3,6$ & $2,4-\mathrm{Me}_{2}-\mathrm{Man}$ & 3.16 \\
33.526 & $1 \rightarrow$ & $2,3,4-\mathrm{Me}_{3}-\mathrm{Xyl}$ & 0.32 \\
\hline
\end{tabular}

methylated sugars, suggesting that the backbone of S-3-1 may be composed of 1,6-linked Galp, 1,4- linked Glcp, and 1,2linked Manp residues, with branches attached to the O-6 of some of the 1,4,6-linked Galp and 1,3,6-linked Manp. This inference also agrees with the results obtained from periodate oxidation and Smith degradation analysis.

$600-\mathrm{MHz}$ NMR spectra of S-3-1 are shown in Figure 4. The ${ }^{1} \mathrm{H}$ and ${ }^{13} \mathrm{C}$-NMR spectra of polysaccharide S-3-1 were crowded in narrow regions ranging from 3.0 to $5.5 \mathrm{ppm}\left({ }^{1} \mathrm{H}\right.$ $\mathrm{NMR})$ and 60 to $110 \mathrm{ppm}\left({ }^{13} \mathrm{C} \mathrm{NMR}\right)$ which were typical of polysaccharides [18]. The ${ }^{1} \mathrm{H}$ NMR signals at 5.0-5.3 ppm and 4.4-5.0 ppm were assigned as the anomeric protons of the alpha-glycosidic configuration and beta-glycosidic configuration [18]. The weak resonances at 1.3 and $1.26 \mathrm{ppm}$ were attributed to the methyl group of Rha [12]. In the anomeric region, the signals at $110.24 \mathrm{ppm}$ were assigned to alpha-Araf, the signals at 105.1 and $98.35 \mathrm{ppm}$ were assigned to beta-Glcp and alpha-Glcp, the signals at 101.27 and $101.24 \mathrm{ppm}$ were assigned to beta-Galp [29], and the signals at 100.51 and 100.31 ppm were assigned to alpha-Manp. The signals for carbons of alpha-Araf and beta-Glc $(1 \rightarrow 6)$ were obviously stronger than those of beta-Galp and Glc [30]; this suggested that Ara and
beta-Glc $(1 \rightarrow 6)$ residues were present in flexible side chains. The chemical shift at $19.4 \mathrm{ppm}$ was assigned to the methyl group of Rha, which was consistent with the detected sugar compositions.

AFM is a powerful tool for directly observing the surface topography or morphology of macromolecule [31, 32] and was used to provide direct evidence of the chain conformation of S-3-1 polysaccharide extracted from SJZD. The S-31 image taken from AFM is shown in Figure 5. The S-31 molecular chain was found to be a spherical distribution polysaccharide with winding coil and multiple nodules held together by intermolecular interactions.

The AFM figure of S-3-1 at different concentrations showed that the morphology of the polysaccharide changed with concentration. At high concentrations of the polysaccharide solution, the molecules wrapped around each other, held together by molecular interatomic forces and van der Waals forces. At low concentrations of the polysaccharide solution, the molecules curled into a globular structure. Figure 5(c) shows the images of the polysaccharide in water, captured at a concentration of $10 \mathrm{ng} / \mathrm{mL}$. As expected, the polysaccharides that existed as flexible chains turned into the branched and knotted state as observed. This indicated that the morphology of the polysaccharide changed with change in the surrounding environment. Hence it was speculated that molecular interatomic forces and van der Waals forces play a major role in the morphology of polysaccharide chains.

3.3. Anticomplement Activities of S-3-1 and S-3-2. The effects of polysaccharide S-3-1 in activation of human complement through the classical pathway were examined in 1:40-diluted NHS, using heparin as a reference and as shown in Table 3. The S-3-1 polysaccharide exhibited inhibitory effects on the classical pathway of the complement system, evidencing $\mathrm{CH}_{50}$ values of $530 \mu \mathrm{g} / \mathrm{mL}$, which was close to the $\mathrm{CH}_{50}$ value of heparin $(500 \mu \mathrm{g} / \mathrm{mL})$. This showed that S-3-1 exhibited inhibitory effects comparable to heparin on the complement system. However, no anticomplement activity was detected for polysaccharide S-3-2. 


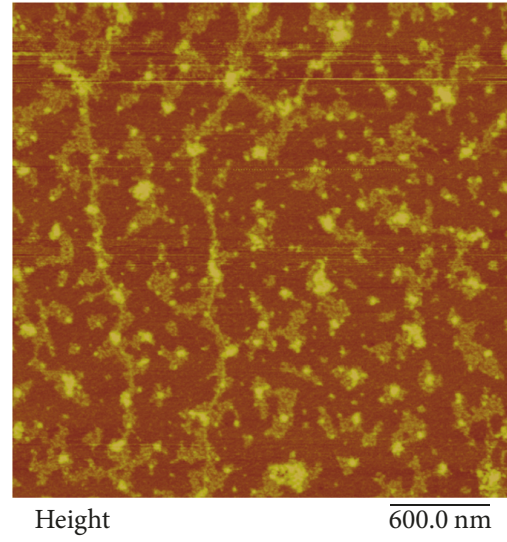

(a)
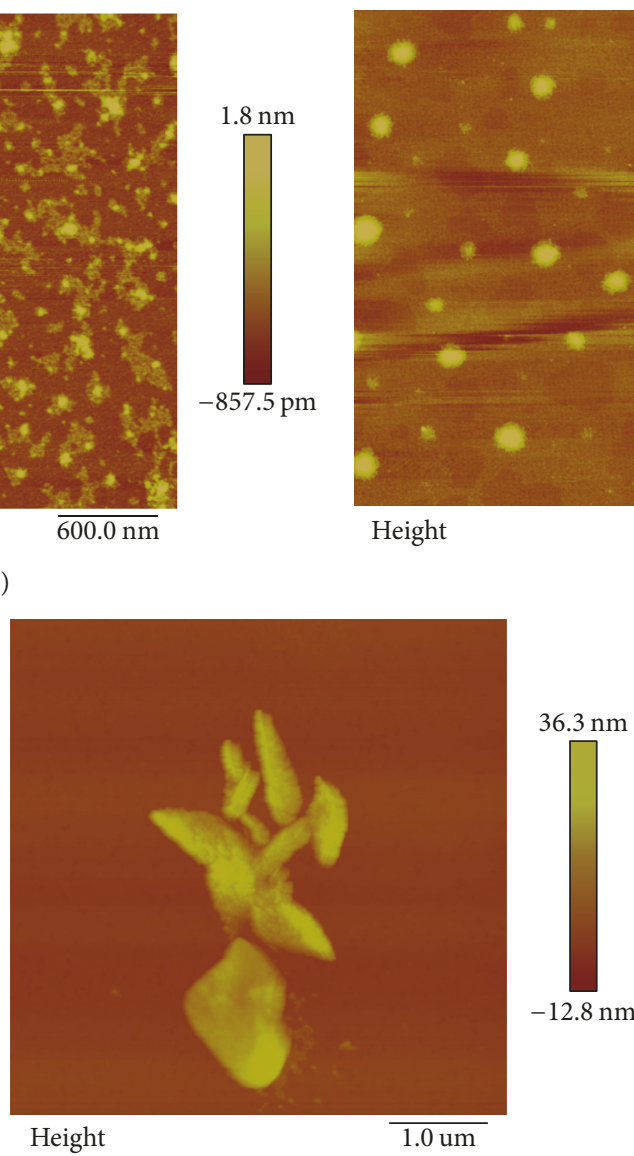

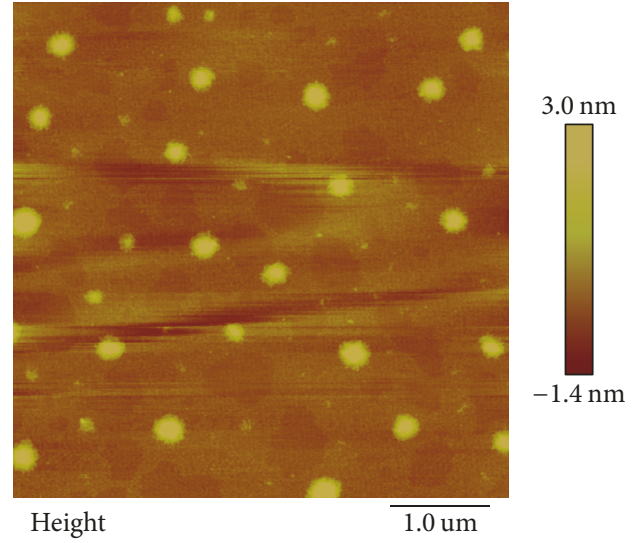

(b)

(c)

Figure 5: AFM topographic image of S-3-1 deposited on mica. (a) At a concentration of $50 \mu \mathrm{g} / \mathrm{ml}$. (b) At a concentration of $1 \mu \mathrm{g} / \mathrm{ml}$. (c) At a concentration of $10 \mathrm{ng} / \mathrm{ml}$.

TABLE 3: Anticomplement activity of two polysaccharides (S-3-1 and S-3-2) isolated from SJZD through the classical pathway.

\begin{tabular}{lc}
\hline Compounds & $\mathrm{CH}_{50}(\mu \mathrm{g} / \mathrm{mL})$ \\
\hline S-3-1 & $530 \pm 20$ \\
S-3-2 & NA \\
Heparin & $500 \pm 17$ \\
\hline
\end{tabular}

Data are expressed as mean \pm SD of triplicate measurements; NA indicated no inhibitory effect. Heparin was used as the positive control.

\section{Discussions and Conclusion}

In this study, S-3, a polysaccharide fraction from SJZD, was purified, and the homogeneous polysaccharide S-31 separated from it. S-3-1 was assayed for immunological activities in vitro and structural characterization. The results showed that S-3-1 is comprised of Rha, Ara, Xyl, Man, Gal, and Glc and mainly contained 1,4-linked-Glc and 1,6-linked-Gal glycosidic bonds. It has been reported that macrophage phagocytosis activity of ginseng is due to a polysaccharide that is a type I rhamnogalacturonan (RG-I) pectin (WGPA-2-RG) with the arabinogalactan side chains [33]. The Atractylodis macrocephalae Koidz polysaccharides are found to be composed of Rha, Ara, Xyl, Man, Glc, and Gal, with a backbone constructed by Glc and the branches constituted by Rha [34]. It has also been reported that polysaccharides extracted from $P$. cocos and fractionated with size-exclusion column chromatography gave a water-soluble 1,6-branched 1,3-alpha-D-Gal structure [35]. Polysaccharides from Liquorice roots have been found to contain about $81 \%$ carbohydrates and $19 \%$ protein, with the polysaccharide content to mainly contain Ara, Gal, Glc, and GlcA [9]. This showed that the polysaccharides isolated from the four crude drugs comprising SJZD were different from S-3-1 which contains six kinds of monosaccharides. Thus, it was speculated that the active polysaccharide of SJZD originated from the decocting process of the four crude drugs.

The complement system is a vital part of the body's immune system and is important for maintaining health. The complement pathway plays an essential role in the host's defense against pathogens [36]. Many active ingredients from TCM have been proved to possess widespread anticomplementary effect [37]. In the present study of natural products, polysaccharide was found to be one of the best complement inhibitors. Our results have shown that the purified polysaccharide S-3-1 displayed a certain inhibitory effect on 
the complement system, with $\mathrm{CH}_{50}$ values of $530 \mu \mathrm{g} / \mathrm{mL}$. It is evident that the homogeneous polysaccharide S-3-1 plays an important role in SJZD's immunologically activities.

In this study, for the first time, the immune active anti-inflammatory mechanism of polysaccharide was proved through anticomplement, and the structure of S-3-1 was characterized as well. Based on review of data from previous literature, it was found that the chemical composition of S-31 and sugar residue connection have not been reported yet, indicating that S-3-1 is produced from the decocted SJZD prescription process rather than from any of the four single herbs. Polysaccharide structure analysis of SJZD will provide a theoretical basis for research on the functions of TCM prescriptions in future.

\section{Conflicts of Interest}

The authors declare that there are no conflicts of interest regarding the publication of this paper.

\section{Acknowledgments}

This work was supported by the National Natural Science Foundation of China (no. 81473318).

\section{References}

[1] Y. Y. Liu, Z. H. Hu, and Y. M. Ma, “The influence of Sijunzi decoction on the pharmacokinetics of levofloxacin in rats with reserpine pretreatment," Asian Pacific Regional International Society for the Study of Xenobiotics Meeting, vol. 40, pp. 97-97.

[2] N. Zhang, S. Guo, H. Li et al., "Effects of sijunzi decoction on small intestinal $\mathrm{T}$ lymphocyte subsets differentiation in reserpine induced spleen deficiency rats," Journal of Animal and Veterinary Advances, vol. 11, no. 9, pp. 1290-1298, 2012.

[3] B. Xiong and H. Qian, "Effects of Sijunzi decoction and Yupingfeng powder on expression of janus kinase-signal transducer and activator of transcription signal pathway in the brain of spleen-deficiency model rats," Journal of Traditional Chinese Medicine, vol. 33, no. 1, pp. 78-84, 2013.

[4] W. Xiu, Z. Lian, L. Xia, D. Xiangliang, W. Ruyan, and W. Junwei, "Effect of polysaccharide of Sijunzi Decoction on gut flora and immune function in spleen-deficiency mice," Pharmacology and Clinics of Chinese Materia Medica, vol. 2, p. 005, 2014.

[5] L. Liu, L. Han, D. Y. L. Wong et al., "Effects of Si-Jun-Zi decoction polysaccharides on cell migration and gene expression in wounded rat intestinal epithelial cells," British Journal of Nutrition, vol. 93, no. 1, pp. 21-29, 2005.

[6] Y. Wang, M. Huang, R. Sun, and L. Pan, "Extraction, characterization of a Ginseng fruits polysaccharide and its immune modulating activities in rats with Lewis lung carcinoma," Carbohydrate Polymers, vol. 127, pp. 215-221, 2015.

[7] R. Wang, G. Zhou, M. Wang, Y. Peng, and X. Li, "The metabolism of polysaccharide from Atractylodes macrocephala Koidz and its effect on intestinal microflora," Evidence-Based Complementary and Alternative Medicine, vol. 2014, Article ID 926381, 7 pages, 2014.

[8] H. Wang, J. F. Mukerabigwi, Y. Zhang et al., "In vivo immunological activity of carboxymethylated-sulfated $(1 \rightarrow 3)$ $\beta$-d-glucan from sclerotium of Poria cocos," International Journal of Biological Macromolecules, vol. 79, pp. 511-517, 2015.
[9] N. Wittschier, G. Faller, and A. Hensel, "Aqueous extracts and polysaccharides from Liquorice roots (Glycyrrhiza glabra L.) inhibit adhesion of Helicobacter pylori to human gastric mucosa," Journal of Ethnopharmacology, vol. 125, no. 2, pp. 218223, 2009.

[10] Z. Cao, J. Wang, B. Wang, R. Rong, Z. Tang, and H. Wang, "Experimental study of anti-tumor activity of complex polysaccharide in vitro and in vivo," Chinese Journal of Basic Medicine in Traditional Chinese Medicine, vol. 16, no. 6, pp. 480-482, 2010.

[11] R. Wang, Y. Peng, H. Meng, and X. Li, "Protective effect of polysaccharides fractions from Sijunzi decoction in reserpineinduced spleen deficiency rats," RSC Advances, vol. 6, no. 65, pp. 60657-60665, 2016.

[12] $\mathrm{H}$. Hu, H. Liang, and $\mathrm{Y}$. Wu, "Isolation, purification and structural characterization of polysaccharide from Acanthopanax brachypus," Carbohydrate Polymers, vol. 127, pp. 94-100, 2015.

[13] M. B. Hall, "Efficacy of reducing sugar and phenol-sulfuric acid assays for analysis of soluble carbohydrates in feedstuffs," Animal Feed Science and Technology, vol. 185, no. 1-2, pp. 94100, 2013.

[14] V. J. Tomazic-Jezic, A. D. Lucas, A. Lamanna, and M. E. Stratmeyer, "Quantitation of natural rubber latex proteins: Evaluation of various protein measurement methods," Toxicology Mechanisms and Methods, vol. 9, no. 3, pp. 153-164, 1999.

[15] Z.-F. Zhang, G.-Y. Lv, T.-T. Song et al., "Comparison of the preliminary characterizations and antioxidant properties of polysaccharides obtained from Phellinus baumii growth on different culture substrates," Carbohydrate Polymers, vol. 132, article no. 9984, pp. 397-399, 2015.

[16] X. Jia, C. Zhang, J. Qiu et al., "Purification, structural characterization and anticancer activity of the novel polysaccharides from Rhynchosia minima root," Carbohydrate Polymers, vol. 132, pp. 67-71, 2015.

[17] K. Soukup, A. Halfmann, M. LeBras et al., "The MAPKactivated kinase MK2 attenuates dendritic cell-mediated Th1 differentiation and autoimmune encephalomyelitis," The Journal of Immunology, vol. 195, no. 2, pp. 541-552, 2015.

[18] Y. Mei, H. Zhu, Q. Hu et al., "A novel polysaccharide from mycelia of cultured Phellinus linteus displays antitumor activity through apoptosis," Carbohydrate Polymers, vol. 124, pp. 90-97, 2015.

[19] I. Ciucanu and F. Kerek, "A simple and rapid method for the permethylation of carbohydrates," Carbohydrate Research, vol. 131, no. 2, pp. 209-217, 1984.

[20] L. Shao, Z. Wu, H. Zhang, W. Chen, L. Ai, and B. Guo, "Partial characterization and immunostimulatory activity of exopolysaccharides from Lactobacillus rhamnosus KF5," Carbohydrate Polymers, vol. 107, no. 1, pp. 51-56, 2014.

[21] D. Flamm and W. Blaschek, "A sulfated cyanobacterial polysaccharide proven as a strong inhibitor of human complement activity in an in vitro assay," Planta Medica, vol. 80, no. 12, pp. 1009-1016, 2014.

[22] Y. Qin, Q. Wen, J. Cao, C. Yin, D. Chen, and Z. Cheng, "Flavonol glycosides and other phenolic compounds from Viola tianshanica and their anti-complement activities," Pharmaceutical Biology, vol. 54, no. 7, pp. 1140-1147, 2016.

[23] P. Wei, Q. Liu, D. Li, Q. Zheng, J. Zhou, and J. Li, "Acute nicotine treatment attenuates lipopolysaccharide-induced cognitive dysfunction by increasing BDNF expression and inhibiting neuroinflammation in the rat hippocampus," Neuroscience Letters, vol. 604, pp. 161-166, 2015. 
[24] B. Qu, J. Cao, F. Zhang et al., "Type i interferon inhibition of microRNA-146a maturation through up-regulation of monocyte chemotactic protein-induced protein 1 in systemic lupus erythematosus," Arthritis \& Rheumatology, vol. 67, no. 12, pp. 3209-3218, 2015.

[25] Q. Wu, H. Qu, J. Jia et al., "Characterization, antioxidant and antitumor activities of polysaccharides from purple sweet potato," Carbohydrate Polymers, vol. 132, Article ID 10034, pp. 31-40, 2015.

[26] Z. Yu, L. Liu, Y. Xu et al., "Characterization and biological activities of a novel polysaccharide isolated from raspberry (Rubus idaeus L.) fruits," Carbohydrate Polymers, vol. 132, Article ID 10059, pp. 180-186, 2015.

[27] D. Qiao, J. Liu, C. Ke, Y. Sun, H. Ye, and X. Zeng, "Structural characterization of polysaccharides from Hyriopsis cumingii," Carbohydrate Polymers, vol. 82, no. 4, pp. 1184-1190, 2010.

[28] L.-J. Jian, J.-M. Chang, M. Ablise, G.-R. Li, and J.-W. He, "Isolation, purification, and structural elucidation of polysaccharides from Alhagi-honey," Journal of Asian Natural Products Research, vol. 16, no. 7, pp. 783-789, 2014.

[29] C. Yang, Y. Gou, J. Chen, J. An, W. Chen, and F. Hu, "Structural characterization and antitumor activity of a pectic polysaccharide from Codonopsis pilosula," Carbohydrate Polymers, vol. 98, no. 1, pp. 886-895, 2013.

[30] S. Zhao, C. Rong, Y. Liu et al., "Extraction of a soluble polysaccharide from Auricularia polytricha and evaluation of its antihypercholesterolemic effect in rats," Carbohydrate Polymers, vol. 122, pp. 39-45, 2015.

[31] L. Shao, Z. Wu, F. Tian et al., "Molecular characteristics of an exopolysaccharide from Lactobacillus rhamnosus KF5 in solution," International Journal of Biological Macromolecules, vol. 72, pp. 1429-1434, 2015.

[32] Z. Huang, Y. Huang, X. Li, and L. Zhang, "Molecular mass and chain conformations of Rhizoma Panacis Japonici polysaccharides," Carbohydrate Polymers, vol. 78, no. 3, pp. 596-601, 2009.

[33] X. Zhang, S. Li, L. Sun et al., "Further analysis of the structure and immunological activity of an RG-I type pectin from Panax ginseng," Carbohydrate Polymers, vol. 89, no. 2, pp. 519-525, 2012.

[34] F. Xie, Y. Li, F. Su, and S. Hu, "Adjuvant effect of Atractylodis macrocephalae Koidz. Polysaccharides on the immune response to foot-and-mouth disease vaccine," Carbohydrate Polymers, vol. 87, no. 2, pp. 1713-1719, 2012.

[35] M.-K. Lu, J.-J. Cheng, C.-Y. Lin, and C.-C. Chang, "Purification, structural elucidation, and anti-inflammatory effect of a watersoluble 1,6-branched 1,3- $\alpha$-d-galactan from cultured mycelia of Poria cocos," Food Chemistry, vol. 118, no. 2, pp. 349-356, 2010.

[36] I. A. Schepetkin, G. Xie, M. A. Jutila, and M. T. Quinn, "Complement-fixing activity of fulvic acid from Shilajit and other natural sources," Phytotherapy Research, vol. 23, no. 3, pp. 373-384, 2009.

[37] H. Wang, H. Wang, S. Shi, J. Duan, and S. Wang, "Structural characterization of a homogalacturonan from Capparis spinosa L. fruits and anti-complement activity of its sulfated derivative," Glycoconjugate Journal, vol. 29, no. 5-6, pp. 379-387, 2012. 

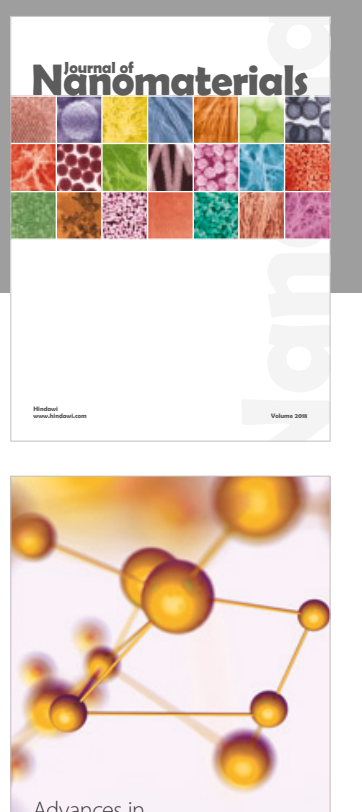

Physical Chemistry
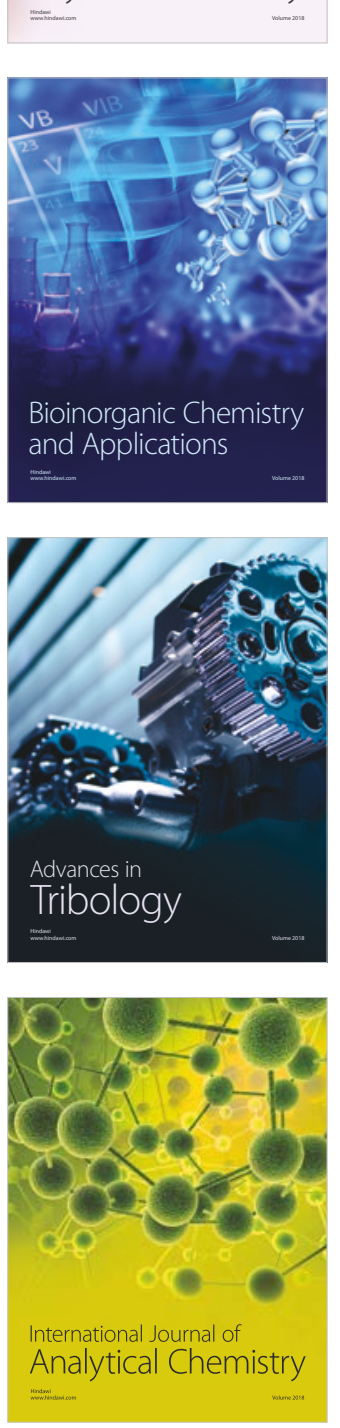

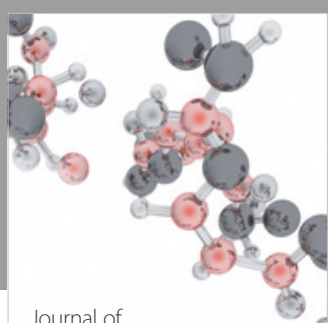

Analytical Methods

in Chemistry

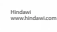

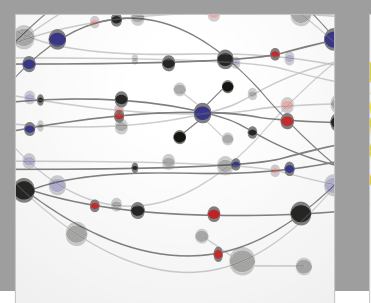

The Scientific World Journal

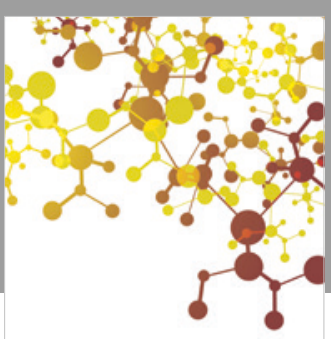

Journal of

Applied Chemistry
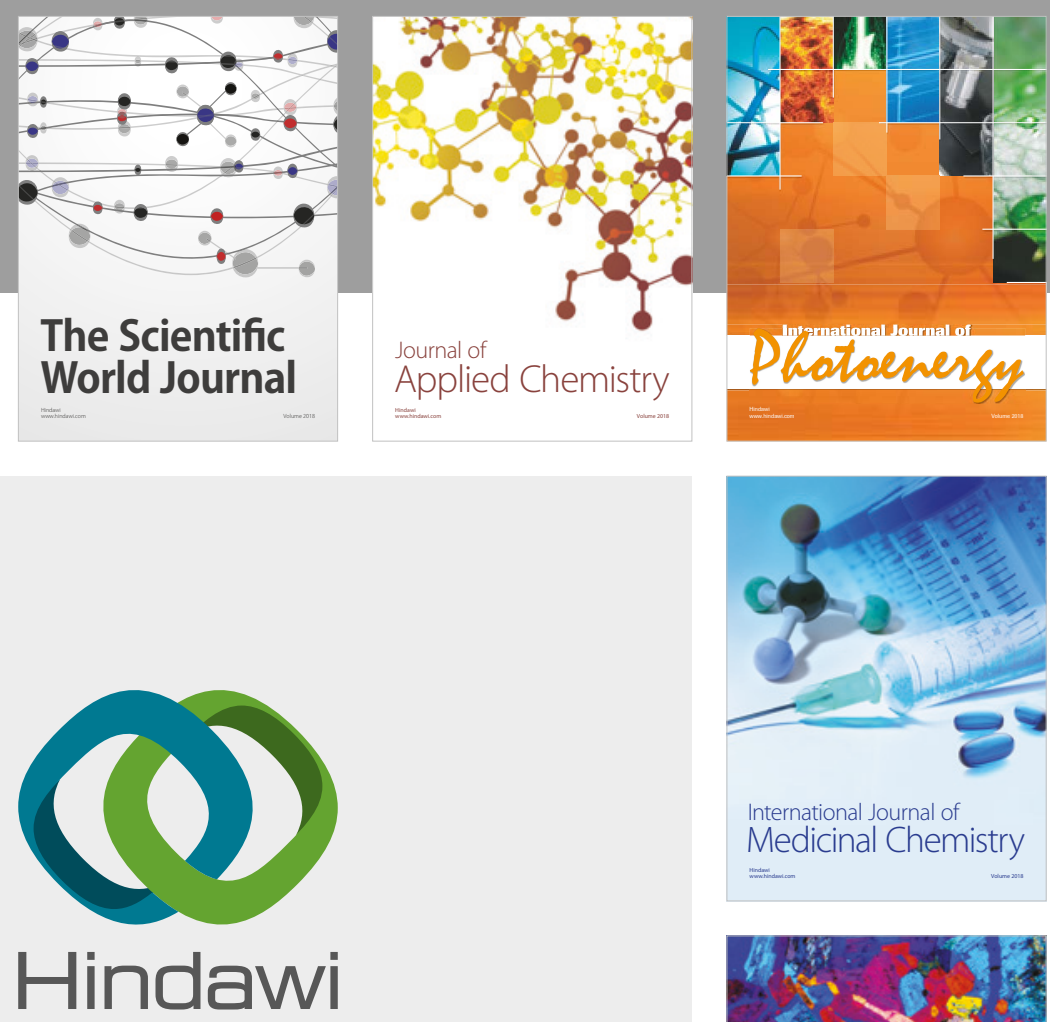

Submit your manuscripts at

www.hindawi.com
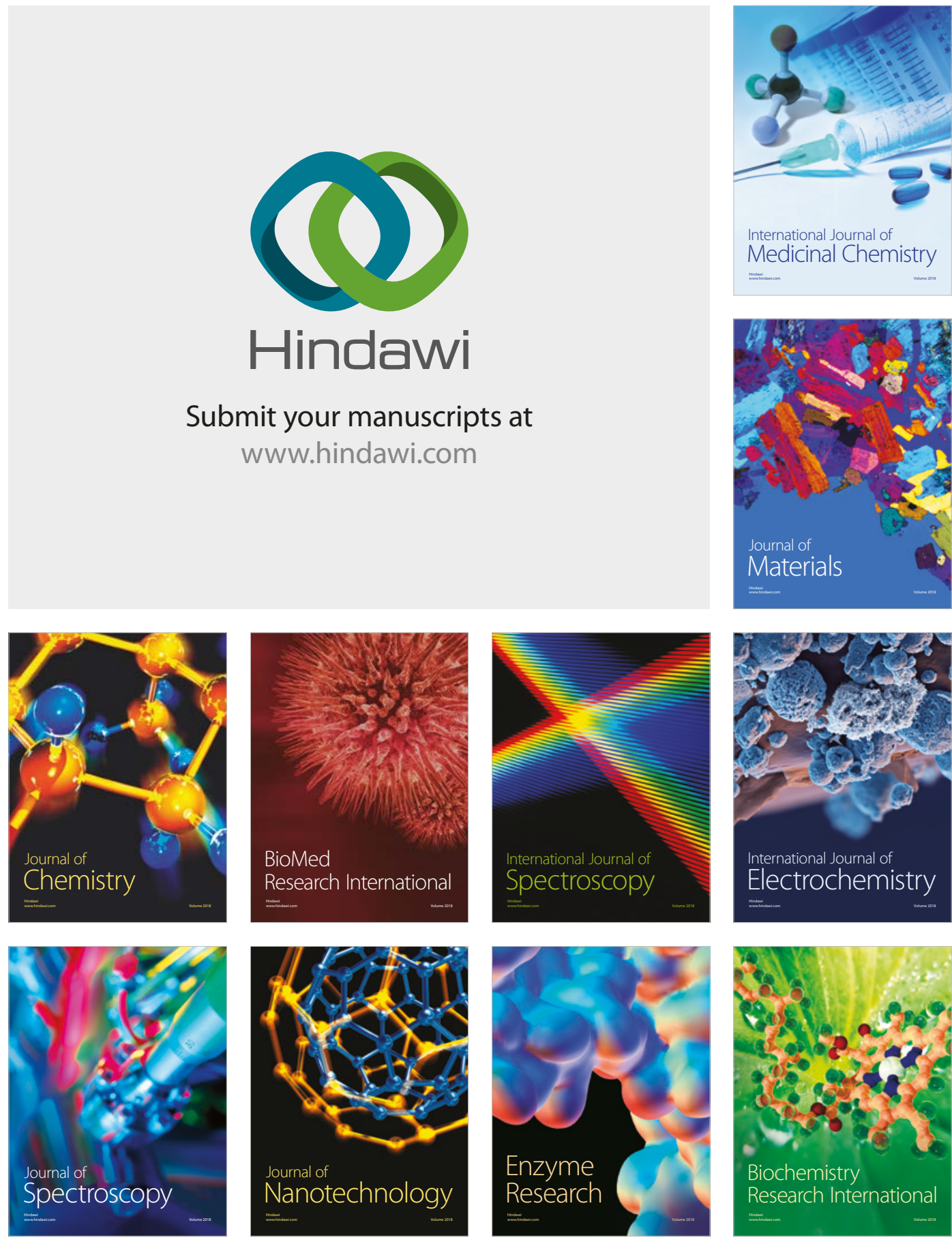
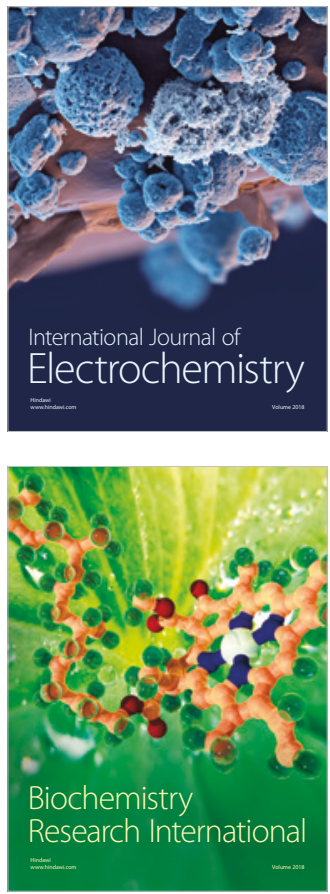